\title{
MÉTODO PARA MONITORAR PERDAS NA APLICAÇÃO DE AGROTÓXICOS NA CULTURA DE TOMATE'
}

\author{
ALDEMIR CHAIM ${ }^{2}$, VERA LUCIA SHERHOLZ SALGADO DE CASTRO ${ }^{3}$, FRANCISCO MIGUEL CORRALES, \\ JOSÉ ABRAHAÃO HADDAD GALVÃO ${ }^{4}$, OSVALDO MACHADO RODRIGUES CABRAL ${ }^{5}$ e GILBERTO NICOLELLA ${ }^{6}$
}

\begin{abstract}
RESUMO - A metodologia disponível para análise de resíduos é muito onerosa para ser usada em estudos de perdas em aplicações de agrotóxicos. Assim, o uso de caldas de pulverização marcadas com traçadores facilmente detectáveis, tem se tornado uma prática amplamente aceita. O objetivo deste trabalho foi avaliar um método de monitoramento das deposições de agrotóxico, caracterizado pelo uso de calda marcada com traçador, e técnica especial de amostragem. Empregou-se o cobre como traçador, na forma de fungicida, cuja análise espectrofotométrica é de baixo custo, fácil execução, além de possibilitar um excelente nível de recuperação. Utilizaram-se cartões de papel mata-borrão como alvo artificial para amostrar as gotas. Cartões grampeados em barbantes, em distâncias preestabelecidas, foram colocados sobre as plantas, possibilitando uma amostragem estratificada por alturas. No solo, os cartões foram colocados sobre placas-de-petri. Para as condições do experimento, o método testado permitiu detectar que, dependendo do porte das plantas, as perdas das aplicações foram da ordem de 59 a $76 \%$.
\end{abstract}

Termos para indexação: método de avaliação de perdas, traçador cúprico, calda de pulverização.

\section{METHOD TO ASSESSMENT THE SPRAYING LOSSES OF PESTICIDE IN TOMATO CROPS}

\begin{abstract}
The available methodology for pesticide residues analysis is very expensive to be used on spraying losses studies and occupational exposure assessment during pesticide application. So, the marking of spray liquids prior to dispersion with easily detectable tracers has become widely accepted practice. The objective of this research was to evaluate a method to assessment the pesticide deposition, characterised by the use of a tracer marked spray liquid and a special sampling technique. The tracer used in this work was the copper, in a fungicide formulation, with low cost and easy atomic absorption spectrophotometric analysis. Cards of blotter-like papers were used as artificial target to catch the droplets. Cards were clipped on cotton strings at constant distances and were disposed on plants possibiliting a height stratified sampling. Cards on Petri dishes are used upon the soil. Between 59 and $76 \%$ of the spray were lost according to plant height.
\end{abstract}

Index terms: loss assessment method, spray liquid, cupric tracer.

\section{INTRODUÇÃO}

Um pesticida precisa ser aplicado em uma áreaalvo particular, ocupada por um inseto, doença ou

\footnotetext{
${ }^{1}$ Aceito para publicação em 17 de agosto de 1998 .

${ }^{2}$ Eng. Agr., M.Sc., Embrapa-Centro Nacional de Pesquisa de Monitoramento e Avaliação de Impacto Ambiental (CNPMA) Caixa Postal 69, CEP 13820-000 Jaguariúna, SP. E-mail: aldemir@cnpma.embrapa.br

${ }^{3}$ Méd. Veterinária, Dra ${ }^{\mathrm{a}}$, Embrapa-CNPMA.

${ }^{4}$ Eng. Agr., Embrapa-CNPMA.

${ }^{5}$ Meteorologista, M.Sc., Embrapa-CNPMA.

${ }^{6}$ Estatístico, M.Sc., Embrapa-CNPMA.
}

erva daninha. A contaminação do meio ambiente por perdas de agrotóxicos para áreas não-alvos tem provocado críticas severas ao uso desses produtos e causado grandes preocupações, quando são noticiados seus efeitos nocivos.

Quando se pulveriza uma cultura, muitas gotas caem entre a folhagem especialmente nos espaços entre as plantas, e atingem o solo. As que se chocam com a folhagem podem coalescer-se com tal intensidade que não conseguem mais ficar retidas, escorrendo para as folhas inferiores, atingindo posteriormente o solo. Esse tipo de pulverização que provoca o escorrimento tem sido recomendado, em muitas ocasiões, para assegurar uma completa cobertu- 
ra da superfície das plantas. Entretanto, uma vez que o escorrimento se inicia, a retenção de químicos pelas folhas é menor do que se a pulverização fosse paralisada, justamente antes de ele ter se iniciado. Nessa modalidade de aplicação, ou alto volume, como tem sido definida, a quantidade do depósito conseguida é proporcional à concentração da calda, mas, independe do volume que é aplicado (Courshee, 1960). Mais de um terço do pesticida aplicado nas culturas pode atingir o solo durante a aplicação. Matthews (1982) tem definido essas perdas como "endoderiva" para diferenciar da "exoderiva", ou seja, para fora da área tratada.

O processo mais empregado para estudar a dinâmica das pulverizações com agrotóxicos tem sido a análise das deposições, com a qual tem se tomado as decisões na escolha de técnicas de aplicação e equipamentos de pulverização. A análise da deposição dos agrotóxicos é baseada na recuperação e detecção das substâncias, da própria superfície das plantas, de alvos artificiais ou por equipamentos específicos de amostragem. A marcação das caldas de pulverização antes da dispersão, com traçadores facilmente detectáveis, tem se tornado uma prática largamente empregada. O custo das análises, a disponibilidade de equipamento analítico e a precisão desejada para os resultados representam fatores importantes na escolha de um determinado traçador para realização dos ensaios. Alguns pesquisadores têm utilizado como traçador os próprios inseticidas (Ware et al., 1969a, 1969b; Ware et al., 1970a, 1970b), partículas fluorescentes como $\mathrm{ZnCaS}$ (Murray \& Vaughan, 1970), agrotóxicos marcados (Maybank et al., 1974), radioisótopos (Dobson et al., 1983), corantes fluorescentes (Liljedahl \& Strait, 1959; Yates \& Akesson, 1963; Fox et al., 1990; Salyani \& Cromwell, 1992) e corantes de alimentos (Pergher et al., 1997). Quantick (1985) cita os principais métodos para análise de recuperação de químicos em experimentos relacionados às técnicas de aplicação. Dentre eles, aquele que utiliza como traçador íons metálicos que podem ser detectados por espectrofotometria de absorção atômica, apresenta ótima sensibilidade e baixo custo de análise. Alguns estudos de deposição utilizando tal metodologia foram conduzidos com fungicidas cúpricos (Herrington et al., 1981; Whitney et al.,
1989; Salyani \& Whitney, 1990; 1991). Entretanto, nesses casos, além de o teor de cobre ter sido determinado colorimetricamente, não foi priorizado pelos autores o relacionamento entre a quantidade de traçador retido nas plantas com o total aplicado.

A grande maioria dos pesquisadores tem preferido fazer uso de substratos artificiais para coleta das gotas, porque nem sempre é possível extrair o traçador dos alvos naturais. Não existem alvos artificiais padrão, podendo variar conforme o tipo do traçador utilizado, o método analítico empregado e sobretudo, a finalidade dos resultados. Entretanto, Davis (1984) apresentou uma série de técnicas para construção de alvos absorventes destinados à avaliação da exposição ocupacional dérmica aos agrotóxicos. Dentre elas, o uso de papel mata-borrão se apresenta como uma opção simples e de baixo custo, que pode ser facilmente adaptada ao estudo de recuperação dos agrotóxicos que atingem as plantas e o solo. Em um estudo de deposição e perdas de agrotóxicos em videira, Pergher et al. (1997) usaram papel absorvente para amostragem do traçador utilizado no experimento.

O objetivo deste trabalho foi testar um método simples para verificar a deposição de agrotóxicos em plantas, utilizando-se calda marcada com traçador em conjunto com técnica de amostragem, de manuseio rápido e baixo custo analítico.

\section{MATERIAL E MÉTODOS}

Foram montados dois ensaios, para verificação da taxa de recuperação de agrotóxicos da cultura de tomate estaqueado, da perda de produto para o solo e do potencial para deriva. Um dos ensaios foi realizado no município de Sumaré, SP, numa área de produção comercial de tomate, onde todo o processo de aplicação foi executado à maneira tradicional da região, com aplicações de grandes volumes de calda. As condições micrometeorológicas do experimento foram medidas por anemômetro mecânico FUESS e um micropsicrômetro de ar forçado, acoplado a um dataloger LI-COR, o que permitiu obter valores da umidade relativa e temperatura, a cada 10 minutos. Iniciou-se a coleta dos dados micrometeorológicos, $30 \mathrm{mi}$ nutos antes das pulverizações e encerrou-se 30 minutos após. Um outro ensaio foi conduzido na área experimental da Embrapa-Centro Nacional de Pesquisa de Monitoramento e Avaliação de Impacto Ambiental, 
Jaguariúna, SP, repetindo-se o mesmo método de aplicação do agricultor. Nesse ensaio, as condições micrometeorológicas foram monitoradas por uma estação Delta T .

Nos dois ensaios utilizou-se pulverizador estacionário com lanças manuais e bicos de pulverização do tipo leque Yamaho D3.

Como traçador foi usado um fungicida cúprico, contendo em sua formulação o equivalente a $350 \mathrm{~g}$ de cobre metálico $/ \mathrm{kg}$. O produto foi aplicado diluído em água procurando-se na medida do possível, o preparo de caldas com teor de $1.400 \mathrm{mg} / \mathrm{L}$ de cobre metálico.

Os ensaios foram realizados em talhões de aproximadamente $168 \mathrm{~m}^{2}$, com 6 linhas de plantio de 28 metros de comprimento. O espaçamento utilizado no talhão de Sumaré foi de $0,65 \times 1,00 \mathrm{~m}$, e em Jaguariúna, de $0,50 \times 1,00 \mathrm{~m}$, perfazendo um total de 258 e 336 plantas, respectivamente.

O delineamento experimental foi inteiramente casualizado, tendo como unidades amostrais as plantas e como tratamentos diferentes alturas de amostragens. A aplicação do traçador foi feita em três estádios de desenvolvimento da cultura e as amostragens foram feitas em: 10,25 e $40 \mathrm{~cm}$, para a cultura com $50 \mathrm{~cm} ; 20,40,60,80$ e $100 \mathrm{~cm}$, cultura com $110 \mathrm{~cm}$; e $25,55,85,115$ e $145 \mathrm{~cm}$, com $160 \mathrm{~cm}$ de altura. A análise estatística foi feita através de teste não-paramétrico de Kuskal-Wallis descrito por Campos (1979).

As amostragens foram realizadas com alvos artificiais constituídos de papel do tipo mata-borrão $-250 \mathrm{~g} / \mathrm{m}^{2}$. Realizou-se um teste para determinar a taxa de retenção de líquido, pesando-se 20 pedaços de papel, de $1,0 \mathrm{~cm}^{2}$, secos e posteriormente encharcados com água; a diferença entre os pesos forneceu a quantidade de líquido retida por $\mathrm{cm}^{2}$ do alvo.

As amostragens nas plantas foram feitas em diferentes alturas com cartões de $10 \times 2 \mathrm{~cm}$, grampeados espaçadamente em barbantes, para facilitar a montagem dos ensaios. Foram distribuídos sobre a superfície de cada planta e de maneira cruzada dois barbantes com cartões, de tal forma que em cada altura de amostragem existissem quatro cartões no mesmo plano. Nas amostragens realizadas nas duas linhas centrais dos talhões, os barbantes com os alvos foram distribuídos aleatoriamente por 3 a 6 plantas de cada linha, num total de 6 a 12 plantas/ talhão, dependendo do ensaio.

Determinou-se a área foliar das plantas, do ensaio de Jaguariúna, com um medidor de área LI-COR LI 3100. A medição foi efetuada em três plantas inteiras, representativas do porte médio do talhão, imediatamente antes de cada teste de aplicação e, com os dados, estabeleceu-se um valor médio de área foliar/planta.
A quantidade de produto que atingiu o solo foi amostrada com 20 cartões de $6 \times 6 \mathrm{~cm}$, acondicionados em placas-de-petri e distribuídos nas duas entrelinhas centrais do talhão.

Para se determinar o resíduo no solo, considerou-se como amostra o cartão disposto em cada ponto amostral. Para as plantas, entretanto, considerou-se a amostra conjunta dos quatro cartões situados num mesmo plano horizontal, ou seja, numa mesma altura. De cada amostra foram retirados 10 discos de $7 \mathrm{~mm}$ de diâmetro, os quais foram colocados numa solução 0,1 normal de ácido clorídrico e submetidos a uma agitação por 30 minutos. O extrato obtido foi filtrado e o teor de cobre determinado em espectrofotômetro de absorção atômica Shimadzu AA 380 . Os teores encontrados nas amostras foram corrigidos pela taxa de extração média das concentrações encontradas e transformados em microgramas de cobre por $\mathrm{cm}^{2}$.

Para calcular a quantidade total de cobre aplicado no talhão foi utilizada a seguinte fórmula:

$\mathrm{D}_{\mathrm{t}}=$ T.V.C,

onde:

$\mathrm{D}_{\mathrm{t}}=$ dose de cobre aplicada no talhão $(\mu \mathrm{g})$;

$\mathrm{T}=$ tempo gasto para pulverizar o talhão $(\mathrm{s})$;

$\mathrm{V}=$ vazão total dos bicos da lança manual (L/s);

$\mathrm{C}=$ concentração de cobre encontrada na calda $(\mu \mathrm{g} / \mathrm{L})$.

Estimou-se a quantidade total do traçador recuperada das plantas do talhão, através da fórmula:

$$
\mathrm{D}_{\mathrm{p}}=\cdot \sum_{\mathrm{i}=1}^{\mathrm{n}} \mathrm{R}_{\mathrm{p}} \mathrm{i} \cdot \mathrm{N} \cdot \mathrm{A} \cdot \mathrm{n}^{-1}
$$

onde:

$\mathrm{D}_{\mathrm{p}}=$ depósito total de cobre encontrados nas plantas do talhão $(\mu \mathrm{g})$;

$\mathrm{n}=$ número de alturas de amostragem;

$\mathrm{R}_{\mathrm{p}}=$ resíduo de cobre encontrado em cada altura $\mathbf{i}$ de amostragem $\left(\mu \mathrm{g} / \mathrm{cm}^{2}\right)$;

$\mathrm{N}$ = número de plantas do talhão;

$\mathrm{A}$ = área foliar média $\left(\mathrm{cm}^{2} /\right.$ planta $)$.

A quantidade de cobre total que atingiu o solo foi estabelecida descontando-se a área coberta pelas plantas, considerando o diâmetro médio da base das copas e a densidade de plantio:

$$
D_{s}=\left[A_{s}-N\left(0,7854 . d_{p}^{2}\right)\right] R_{s}
$$

onde:

$\mathrm{D}_{\mathrm{s}}=$ depósito total de cobre estimado para o solo $(\mu \mathrm{g}) ;$

$\mathrm{A}_{\mathrm{s}}=$ área total do solo do talhão $\left(\mathrm{cm}^{2}\right)$;

$\mathrm{N}$ = número de plantas do talhão;

$\mathrm{d}_{\mathrm{p}}=$ diâmetro médio da base da copa das plantas $(\mathrm{cm})$; 
$\mathrm{R}_{\mathrm{s}}=$ resíduo médio de cobre encontrado nas amostras coletadas sobre a superfície do solo $\left(\mu \mathrm{g} / \mathrm{cm}^{2}\right)$.

Os dados percentuais de cobre depositados nas plantas e no solo, bem como aquele perdido por exoderiva, foram calculados em relação ao total aplicado.

\section{RESULTADOS E DISCUSSÃO}

O fungicida cúprico utilizado nos experimentos tanto em Sumaré quanto Jaguariúna era constituído de um sal - oxicloreto de cobre - muito estável nas condições de campo e que não exigiu nenhum cuidado com refrigeração de amostra ou proteção contra radiação. A estabilidade química do traçador é um fator de extrema importância porque, dependendo do seu grau de degradação no ambiente, é necessário programar o ensaio para que a coleta das amostras seja realizada em tempo muito curto. Problemas de fotodegradação com traçadores fluorescentes foram relatados por Fox et al. (1990) e Salyani \& Cromwell (1992). A perda com fotodegradação do corante de alimentos (tartrazina), utilizado como traçador por Pergher et al. (1997), foi de 8,8\%, após exposição à radiação solar de $800 \mathrm{~W} / \mathrm{m}^{2}$, durante 30 minutos.

A taxa de recuperação de cobre pelo método analítico empregado foi em média de $96 \%$ para as concentrações encontradas nos experimentos e, neste caso, os valores dos resíduos originalmente obtidos foram corrigidos por esse fator.

Quanto ao alvo de amostragem utilizado no experimento, deve ser considerado que a taxa de retenção máxima de água pelo papel mata-borrão, foi de $0,0442 \mathrm{~g} / \mathrm{cm}^{2}$. O resíduo máximo de cobre que poderia ser encontrado no papel estaria em torno de $61,88 \mu \mathrm{g} / \mathrm{cm}^{2}$ considerando a sua capacidade de retenção e a concentração da calda $(1.400 \mathrm{mg} / \mathrm{L})$. A superfície das folhas do tomateiro, provavelmente, não apresenta uma taxa de retenção de líquido superior à do papel mata-borrão, pois os teores de cobre encontrados nas análises indicam que não houve saturação em nenhuma amostra.

Os resultados encontrados na Tabela 1 mostram que os resíduos encontrados no solo são relativamente altos e, na maioria dos casos, superiores àqueles encontrados nas plantas. Nesse caso deve ser considerado que quanto menor o porte da cultura, tanto maior será a área do solo exposta. Isso indica a necessidade de se alterar a forma de aplicação, não só por uma diminuição no número de bicos da lança, como pela escolha de outros bicos e pressões de trabalho que proporcionem uma redução dessas perdas. Pode ser observado, também, que não existe relação entre volume aplicado e resíduo de produto na planta, o que corrobora as informações de Courshee (1960).

A técnica de amostragem com papel mata-borrão, grampeado em barbante em distância predeterminada, agilizou os ensaios de campo, fornecendo dados que podem ser usados para verificar a qualidade da deposição dos agrotóxicos nas plantas. Essa técnica pode ser utilizada em estudos mais refinados, para verificar qual é a distribuição dos agrotóxicos nas plantas. Na Tabela 2, a análise estatística dos dados comprova que houve diferença significativa de deposição em diferentes alturas das plantas, no ensaio de Sumaré. Tal comportamento não se verificou no de Jaguariúna, e pode estar relacionado às diferentes velocidades de aplicação e vazões dos bicos utilizados nos dois locais. As menores velocidades de aplicação empregadas em Jaguariúna (Tabela 2), contribuíram para uma cobertura mais uniforme das plantas. $\mathrm{O}$ fato de ter sido escolhido um teste não-paramétrico para análise estatística, é justificado pelo número excessivo de amostras que um teste paramétrico exigiria para atender a alguns pressupostos, tais como normalidade dos dados e precisão desejada. Neste trabalho foram realizadas aproximadamente 800 análises, número esse que subiria para 3.200 caso fosse feito, por exemplo, um delineamento em blocos casualizado, com quatro repetições.

Em virtude da rapidez com que as plantas de tomate se desidratam, não foi possível medir a área foliar na cultura de Sumaré e isso inviabilizou o cálculo das perdas no ensaio. Em Jaguariúna, ao contrário, a quantidade de agrotóxico retido na superfície das plantas pôde ser estimada pela correlação entre a área foliar e a média entre os resíduos encontrados nas diferentes alturas de amostragem. Considerando apenas a quantidade de traçador retido nas plantas, observa-se na Tabela 3 perdas acentuadas, que variam de $59 \%$ em plantas com maior porte, a $76 \%$ nas de menor. Isso é conseqüência do 
TABELA 1. Características dos ensaios e valores de resíduo do traçador encontrados em plantas e solo.

\begin{tabular}{|c|c|c|c|c|c|c|}
\hline \multirow[t]{3}{*}{ Características dos ensaios } & \multicolumn{3}{|c|}{ Jaguariúna } & \multicolumn{3}{|c|}{ Sumaré } \\
\hline & \multicolumn{3}{|c|}{ Altura das plantas $(\mathrm{cm})$} & \multicolumn{3}{|c|}{ Altura das plantas $(\mathrm{cm})$} \\
\hline & 50 & 110 & 160 & 50 & 110 & 160 \\
\hline Velocidade do vento $(\mathrm{m} / \mathrm{s})$ & 2,2 & 3,5 & 3,1 & n.d. ${ }^{1}$ & n.d. & 1,5 \\
\hline Umidade relativa $(\%)$ & 43 & 60 & 42 & n.d. & n.d. & 77 \\
\hline Temperatura $\left({ }^{\circ} \mathrm{C}\right)$ & 28,2 & 29,4 & 25,3 & n.d. & n.d. & 20,8 \\
\hline Velocidade de aplicação $(\mathrm{m} / \mathrm{s})$ & 0,40 & 0,32 & 0,32 & 0,78 & 0,60 & 0,40 \\
\hline Pressão de trabalho $(\mathrm{kPa})$ & 410 & 410 & 410 & 1040 & 1040 & 1040 \\
\hline Vazão da barra de bicos (mL/s) & 23 & 33 & 33 & 25 & 53 & 51 \\
\hline Volume de calda gasto (L/ha) & 523 & 1031 & 1031 & 326 & 884 & 1275 \\
\hline Área foliar/planta $\left(\mathrm{m}^{2}\right)$ & 0,25 & 0,80 & 1,30 & n.d. & n.d. & n.d. \\
\hline Resíduo médio nas plantas $\left(\mu \mathrm{g} / \mathrm{cm}^{2}\right)$ & 3,5 & 3,2 & 2,3 & 2,9 & 3,7 & 2,8 \\
\hline Resíduo médio no solo $\left(\mu \mathrm{g} / \mathrm{cm}^{2}\right)$ & 3,9 & 5,0 & 7,0 & 3,4 & 2,7 & 4,0 \\
\hline
\end{tabular}

TABELA 2. Resíduo de cobre encontrado em diferentes alturas de amostragem, sob três estádios de desenvolvimento da cultura ${ }^{1}$.

\begin{tabular}{lccc}
\hline \multirow{2}{*}{$\begin{array}{l}\text { Altura das } \\
\text { plantas }(\mathrm{cm})\end{array}$} & $\begin{array}{c}\text { Altura das } \\
\text { amostras }(\mathrm{cm})\end{array}$ & \multicolumn{2}{c}{ Local dos ensaios } \\
\cline { 2 - 4 } & Jaguariúna & Sumaré $^{3}$ \\
\hline $50^{3}$ & 25 & $3,2 \mathrm{a}$ & - \\
& 30 & $3,5 \mathrm{a}$ & $1,6 \mathrm{~b}$ \\
& 40 & $3,8 \mathrm{a}$ & $4,3 \mathrm{a}$ \\
\hline $110^{4}$ & 20 & $3,0 \mathrm{a}$ & $2,5 \mathrm{a}$ \\
& 40 & $2,8 \mathrm{a}$ & $3,0 \mathrm{a}$ \\
& 60 & $2,9 \mathrm{a}$ & $3,5 \mathrm{ab}$ \\
& 80 & $3,5 \mathrm{a}$ & $3,8 \mathrm{ab}$ \\
& 100 & $3,9 \mathrm{a}$ & $5,7 \mathrm{a}$ \\
\hline $160^{5}$ & 25 & $3,3 \mathrm{a}$ & $2,0 \mathrm{~b}$ \\
& 55 & $2,0 \mathrm{a}$ & $2,2 \mathrm{~b}$ \\
& 85 & $1,8 \mathrm{a}$ & $2,7 \mathrm{ab}$ \\
& 115 & $1,8 \mathrm{a}$ & $3,5 \mathrm{ab}$ \\
& 145 & $2,4 \mathrm{a}$ & $3,7 \mathrm{a}$ \\
\hline
\end{tabular}

${ }^{1}$ Resíduo expresso em $\mu \mathrm{g} / \mathrm{cm}^{2}$.

2 Teste estatístico aplicado para cada ensaio; médias seguidas pela mesma letra não diferem entre si.

${ }^{3}$ Usou-se o teste de Wilcoxon com nível de significância $\alpha<0,004$.

${ }^{4}$ Nível de significância $\alpha=0,01$.

${ }^{5}$ Nível de significância $\alpha=0,05$.

alto volume de calda aplicada (Courshee, 1960), bem como da relação da deposição com a área foliar, conforme foi demonstrado nos estudos realizados por Pergher \& Gubiani (1995) e Pergher et al. (1997).
Uma comparação grosseira entre técnicas de aplicação pode ser estabelecida quando se compara os resultados de deposição obtidos por Pergher et al. (1997), que utilizaram dois tipos de pulverizadores com ventiladores axiais e um com ventilador centrífugo, com a finalidade de assoprar as gotas na direção das folhas das plantas. Todos os equipamentos depositaram acima de $64 \%$ de agrotóxico na cultura de videira, com máximo desenvolvimento vegetativo. A deposição máxima obtida na cultura do tomate foi de apenas $41 \%$ (Tabela 3). A diferença de deposição nas duas culturas é conseqüência do vento criado pelos ventiladores dos equipamentos utilizados na videira, que além de melhorar a distribuição das gotas aumenta a eficiência da penetração do agrotóxico entre as folhas das plantas. $\mathrm{Na}$ técnica de aplicação empregada na pulverização do tomateiro, as gotas são arremessadas unicamente pela força da pressão hidráulica presente no bico. Teoricamente, em cada passada do aplicador, as gotas deveriam atingir a lateral das plantas da linha mais próxima da pulverização e a lateral das plantas da linha adjacente. Entretanto, a força com que as gotas são arremessadas não é suficiente para que elas atinjam a linha de plantio adjacente, e assim somente uma lateral das plantas recebe uma deposição mais expressiva. Para resolver o problema da penetração das gotas, a maioria dos agricultores eleva a pressão de pulverização, mas isso acrescenta uma série de efeitos colaterais, pois aumenta da exposição dos aplicadores, a perda por deriva e evaporação. 
TABELA 3. Distribuição percentual de agrotóxico, estimada para a cultura de tomate cultivada nos campos experimentais de Jaguariúna.

\begin{tabular}{cccc}
\hline $\begin{array}{c}\text { Altura das } \\
\text { plantas } \\
(\mathrm{cm})\end{array}$ & $\begin{array}{c}\text { Produto } \\
\text { retido nas } \\
\text { plantas }\end{array}$ & $\begin{array}{c}\text { Produto } \\
\text { perdido } \\
\text { para o solo }\end{array}$ & $\begin{array}{c}\text { Produto perdido } \\
\text { para local } \\
\text { desconhecido }\end{array}$ \\
\hline 50 & 24 & 39 & 37 \\
110 & 35 & 20 & 45 \\
160 & 41 & 29 & 30 \\
\hline
\end{tabular}

Os resultados obtidos com a validação, em campo, de um método de monitoramento de perdas de agrotóxico demonstram que existe um potencial muito grande para reduzir as doses atualmente empregadas para controlar pragas e doenças na cultura de tomate estaqueado.

\section{CONCLUSÕES}

1. O método de avaliação de perdas, constituído de um sistema de amostragem que utiliza cartões de papel mata-borrão, grampeados em barbantes em distâncias preestabelecidas, agiliza a montagem de ensaios no campo e permite uma análise de deposição estratificada por altura.

2. Fungicidas cúpricos, utilizados como traçadores para avaliação de perdas ou deposição, são estáveis e facilmente analisados através de espectrofotômetro de absorção atômica.

3. Os resultados da validação do método de avaliação de deposição indicam que as perdas na aplicação de agrotóxicos na cultura de tomate estaqueado estão entre 59 a $76 \%$.

\section{AGRADECIMENTOS}

Ao técnico Edenir R. Pereira Filho, pela realização das análises do fungicida cúprico, e à Maria Amélia T. Leme, pelo auxílio na elaboração do texto e referências.

\section{REFERÊNCIAS}

CAMPOS, H. Estatística experimental não paramétrica. Piracicaba: USP-ESALQ, 1979.343p.
COURSHEE, R.J. Some aspects of the application of insecticides. Annual Review of Entomology, Palo Alto, v.5, p.327-352, 1960 .

DAVIS, J.E. Procedures for dermal and inhalation studies to assess occupational exposure to pesticides. In: SIEWIERSKI, M. Determination and assessment of pesticide exposure. Amsterdam: Elsevier, 1984. p.123-131.

DOBSON, C.M.; MINSKI, M.J.; MATTHEWS, G.A Neutron activation analysis using dysprosium as a tracer to measure spray drift. Crop Protection, Oxford, v.2, n.3, p.345-352, 1983.

FOX, R.D.; BRAZZE, R.D.; REICHARD, D.L.; HALL, F.R. Downwind residue from air spraying of a dwarf apple orchard. Transactions of the ASAE, St. Joseph, v.33, n.4, p.1104-1108, 1990.

HERRINGTON, P.J.; MAPOTHER, H.R.; STRINGER, A. Spray retention and distribution on apple trees. Pesticide Science, Oxford, v.12, n.5, p.515-520, 1981.

LILJEDAHL, L.A.; STRAIT, J. Spray deposits measured rapidly. Agricultural Engineering, St. Joseph, v.40, n.6, p.332-335, 1959.

MATTHEWS, G.A. Pesticide application methods. New York: Longman, 1982. 336p.

MAYBANK, J.; YOSHIDA, K.; GROVER, R. Droplet size spectra, drift potential, and ground deposition pattern of herbicide sprays. Canadian Journal of Plant Science, Ottawa, v.54, p.541-546, 1974.

MURRAY, J.A.; VAUGHAN, L.M. Measuring pesticide drift at distance to four miles. Journal of Applied Meteorology, Boston, v.9, p.79-84, 1970.

PERGHER, G.; GUBIANI, R. The effect of spray application rate on foliar deposition in a hedgerow vineyard. Journal of Agricultural Engineering Research, London, n.61, p.205-216, 1995.

PERGHER, G.; GUBIANI, R.; TONETTO, G. Foliar deposition and pesticide losses from three airassisted sprayers in a hedgerow vineyard. Crop Protection, Oxford, v.16, n.1, p.25-33, 1997.

QUANTICK, H.R. Aviation in crop protection, pollution and insect control. London: Collins, 1985. $427 \mathrm{p}$.

SALYANI, M.; CROMWELL, R.P. Spray drift from ground and aerial applications. Transactions of the ASAE, St. Joseph, v.35, n.4, p.1113-1129, 1992. 
SALYANI, M.; WHITNEY, J.D. Effect of oscillators on deposition characteristics of an airblast sprayer. Transactions of the ASAE, St. Joseph, v.34, n.4, p.1618-1622, 1991

SALYANI, M.; WHITNEY, J.D. Ground speed effect on spray deposition inside citrus trees. Transactions of the ASAE, St. Joseph, v.33, n.2, p.361-366, 1990.

WARE, G.W.; APPLE, E.J.; CAHILL, W.P.; GERHARDT, P.D.; FROST, K.R. Pesticide drift. II. Mist-blower vs. aerial application of sprays. Journal of Economic Entomology, College Park, v.62, n.4, p.844-846, 1969a.

WARE, G.W.; APPLE, E.J.; CAHILL, W.P.; GERHARDT, P.D.; FROST, K.R. Pesticide drift. III. Drift reduction with spray thickeners. Journal of Economic Entomology, College Park, v.63, n.6, p.1314-1316, 1970a.

WARE, G.W.; CAHILL, W.P.; GERHARDT, P.D.; WITT, J.M. Pesticide drift. IV. On target deposits from aerial application of insecticides. Journal of Economic Entomology, College Park, v.63, n.6, p.1982-1983, 1970b.

WARE, G.W.; ESTESEN, G.J.; CAHILL, W.P.; GERHARDT, P.D.; FROST, K.R. Pesticide drift. I. High clearance vs. aerial application of sprays. Journal of Economic Entomology, College Park, v.62, n.4, p.840-843, 1969 b.

WHITNEY, J.D.; SALYANI, M.; CHURCHILL, D.B.; KNAPP, J.L.; WHITESIDE, J.O.; LITTELL, R.C. A field investigation to examine the effects of sprayer type, ground speed, and volume rate on spray deposition in Florida citrus. Journal of Agricultural Engineering Research, London, v.42, p.275-283, 1989.

YATES, W.E.; AKESSON, N.B. Fluorescent tracers for quantitative microresidue analysis. Transactions of the ASAE, St. Joseph, v.6, n.2, p.104-107, 114, 1963. 